\title{
Simulation du comportement dynamique d'un système usinant : modélisation de l'interaction outil/matière en présence d'une pièce flexible
}

\author{
Philippe Loronga ${ }^{a}$ Gérard Coffignal et Stéphanie Cohen-Assouline \\ Laboratoire de Mécanique des Systèmes et des Procédés, UMR CNRS 8106, École Nationale Supérieure d'Arts et Métiers, \\ 151 boulevard de l'Hôpital, 75013 Paris, France
}

Reçu le 25 mai 2007, accepté le 24 janvier 2008

\begin{abstract}
Résumé - La simulation du comportement dynamique d'un système usinant conduit à la résolution d'un problème non-linéaire de vibrations où la modélisation de l'interaction outil/pièce joue un rôle central. Cette interaction a pour particularité d'évoluer au cours du temps suite à une modification régulière de la frontière du domaine pièce qui correspond à l'enlèvement progressif de matière. La flexibilité de la pièce complique très nettement son évaluation. L'objectif principal de ce travail est de proposer une démarche permettant de faire évoluer, tout au long de l'usinage, le modèle géométrique décrivant la surface usinée dans un contexte de pièce déformable. Les différents modèles nécessaires à la simulation du comportement dynamique du système usinant sont présentés ainsi que la démarche adoptée pour modéliser l'interaction outil/pièce. Deux exemples simples montrent l'intérêt de la méthode.
\end{abstract}

Mots clés : Usinage / vibrations / état de surface / simulation numérique / procédé de fabrication

\begin{abstract}
Simulation of the dynamical behaviour of a machining system: modeling of the tool/workpiece interaction with flexible workpiece. The simulation of a machining system leads to the resolution of a non-linear problem of vibrations where the modeling of the interaction tool-part plays a central part. This interaction has as a characteristic to evolve in the course of time following a regular modification of the domain boundary of the part which corresponds to the progressive removal of matter. The flexibility of the part complicates its evaluation very clearly. It is the main objective of this work to give a significant contribution to the way of solving the problem in that case. The various models necessary to the simulation of the dynamic behavior of the machining system are presented as well as the approach adopted to model the interaction toolpart. Two simple examples show the interest of the method.
\end{abstract}

Key words: Machining / vibrations / surface quality / numerical simulation / manufacturing process

\section{Introduction}

Lors de l'usinage d'une pièce dont le comportement ne peut pas être considéré comme parfaitement rigide, la prédiction du comportement dynamique du système usinant (système Pièce/Outil/Machine : système $\boldsymbol{P O M}$ ), présente un grand intérêt. Elle est indispensable pour pouvoir déterminer a priori la faisabilité d'une opération d'usinage en fonction de la précision attendue et des diverses contraintes à respecter.

Actuellement, l'absence de logiciels commerciaux permettant ces simulations est un obstacle à la mise en

\footnotetext{
a Auteur pour correspondance :

philippe.lorong@paris.ensam.fr
}

place de chaînes numériques complètes de simulation des procédés de fabrication dans les entreprises. Le travail présenté ici a pour objectif de contribuer à combler cette lacune en proposant des modèles permettant de réaliser des simulations, y compris lorsque la pièce est flexible et qu'elle est le siège de vibrations induites par l'usinage. Ces simulations imposent de résoudre des problèmes de dynamique non-linéaire où la modélisation de l'interaction outil/pièce joue un rôle central. Le mouvement relatif outil/pièce ainsi que la modification régulière de la surface usinée font que cette interaction ne cesse d'évoluer au cours du temps. Le mouvement relatif outil/pièce résulte conjointement de la trajectoire théorique outil/pièce prescrite, fixant le mouvement relatif des référentiels $\mathcal{R}_{\text {outil }}$ par rapport au référentiel $\mathcal{R}_{\text {pièce }}$, et des vibrations et 


\section{Nomenclature}

\begin{tabular}{|c|c|}
\hline${ }^{*} \mathcal{B}$ & domaine contenant avec ${ }^{*} \Omega$ initial ou confondu avec lui \\
\hline${ }^{\mathrm{r}} \mathcal{B}$ & image de ${ }^{*} \mathcal{B}$ dans la configuration matérielle de référence \\
\hline${ }^{t} \mathcal{B}$ & image de ${ }^{*} \mathcal{B}$ dans la configuration actuelle \\
\hline${ }^{*} \mathcal{B}^{+}$ & extension de ${ }^{*} \mathcal{B}$ \\
\hline${ }^{\mathrm{r}} \mathcal{B}^{+}$ & image de ${ }^{*} \mathcal{B}^{+}$dans la configuration matérielle de référence \\
\hline${ }^{t} \mathcal{B}^{+}$ & image de ${ }^{*} \mathcal{B}^{+}$dans la configuration actuelle \\
\hline $\mathrm{C}$ & matrice d'amortissement et/ou d'effets gyroscopiques \\
\hline${ }^{\mathrm{r}} E_{3}$ & espace matériel de référence \\
\hline$E_{3}$ & espace réel \\
\hline$f_{\mathrm{d}}$ & fréquence de passage des dents \\
\hline$f_{1}$ & première fréquence propre de la pièce \\
\hline$f_{\text {coupe }}$ & densité linéique d'effort de coupe \\
\hline $\mathrm{G}_{\text {calcul }}^{\text {hexa }}$ & matrice de liaison entre $\boldsymbol{q}$ et $\boldsymbol{q}_{\text {hexa }}$ \\
\hline$h_{\text {coupe }}$ & épaisseur locale instantanée de coupe \\
\hline $\mathrm{K}$ & matrice de rigidité \\
\hline M & matrice de masse \\
\hline $\mathcal{M}_{\mathrm{b}}$ & modèle $\boldsymbol{B R} \boldsymbol{E P}$ des domaines balayés par les faces de coupe \\
\hline$n_{\text {int }}$ & nombre de sous-incréments pour les calculs de géométrie \\
\hline $\mathrm{N}\left({ }^{*} X\right)$ & $\begin{array}{l}\text { matrice des fonctions d'interpolation, } \\
\text { maillage pièce, calculs mécaniques }\end{array}$ \\
\hline $\mathrm{N}_{\text {hexa }}\left({ }^{*} X\right)$ & $\begin{array}{l}\text { matrice des fonctions d'interpolation, } \\
\text { maillage hexaédrique, calculs de géométrie }\end{array}$ \\
\hline$P O M$ & système Pièce/Outil/Machine \\
\hline$p$ & colonne des paramètres de géométrie du système $P O M$ \\
\hline$Q_{\text {coupe }}$ & colonne des efforts généralisés (due aux efforts de coupe) \\
\hline$q$ & colonne des paramètres cinématiques du maillage de la pièce \\
\hline$q_{\text {hexa }}$ & colonne des paramètres cinématiques du maillage hexaédrique \\
\hline $\mathcal{R}_{\text {pièce }}$ & référentiel pièce (référentiel de $E_{3}$ ) \\
\hline $\mathcal{R}_{\text {outil }}$ & référentiel outil mobile par rapport à $\mathcal{R}_{\text {pièce }}$ \\
\hline$s$ & point $\operatorname{de}^{\mathrm{r}} \mathcal{B}$ \\
\hline$t$ & temps \\
\hline$\Delta t$ & incrément de temps \\
\hline$T_{\mathrm{j}}$ & piquet de temps \\
\hline${ }^{t} U$ & champ de déplacements pour aller de ${ }^{*} \mathcal{B}$ à ${ }^{t} \mathcal{B}$ \\
\hline${ }^{*} X$ & point de ${ }^{*} \mathcal{B}$ \\
\hline${ }^{t} X$ & point de ${ }^{t} \mathcal{B}$ \\
\hline$Z$ & axe dans ${ }^{\mathrm{r}} E_{3}$ parallèle aux supports des Dexels \\
\hline${ }^{t} \Phi$ & application bijective allant $\operatorname{de}^{\mathrm{r}} \mathcal{B}$ à ${ }^{t} \mathcal{B}$ \\
\hline${ }^{*} \Phi$ & application bijective allant $\mathrm{de}^{\mathrm{r}} \mathcal{B}$ à ${ }^{*} \mathcal{B}$ \\
\hline${ }^{t} \Phi^{+}$ & prolongement $\mathrm{de}^{t} \Phi$ \\
\hline$\psi$ & colonne du déséquilibre des efforts généralisés \\
\hline$\Omega$ & vitesse de rotation de la broche \\
\hline${ }^{*} \Omega$ & $\begin{array}{l}\text { domaine occupé par la pièce, dans la } \\
\text { configuration non bridée fixée isostatiquement }\end{array}$ \\
\hline${ }^{\mathrm{r}} \Omega$ & domaine occupé par la pièce, configuration matérielle de référence \\
\hline & domaine occupé par la pièce, configuration actuelle \\
\hline$\partial \Omega_{\text {coupe }}$ & section de coupe instantannée \\
\hline
\end{tabular}

déformations de l'outil et/ou de la pièce. Il est à noter que la surface usinée ne correspond généralement qu'à une fraction réduite de la frontière du domaine de la pièce.

La flexibilité de la pièce complique très nettement l'évaluation de l'interaction outil/pièce ainsi que le suivi de la frontière du domaine qu'elle occupe, à cause de ses vibrations qui ne peuvent pas être négligées. L'effet retard qui provient de l'histoire de l'enlèvement de matière peut de plus conduire à l'auto-excitation du système et être responsable du phénomène de broutement [1]. Ce phénomène de broutement, est fréquemment observé lors de l'usinage de pièces flexibles (carter, voiles minces). Notre démarche consiste à mettre en œuvre une simulation de l'usinage en présence de pièce flexible afin de prédire le broutement dans un tel contexte et de pouvoir tester d'autres configurations d'usinage pour y remédier.

Dans cet article, après avoir rapidement rappelé les différents modèles nécessaires à la simulation du comportement dynamique du système $\boldsymbol{P O M}$, nous précisons la démarche que nous avons adoptée pour modéliser l'interaction outil/pièce lorsque cette dernière est flexible, le schéma incrémental et les schémas itératifs mis en place. 


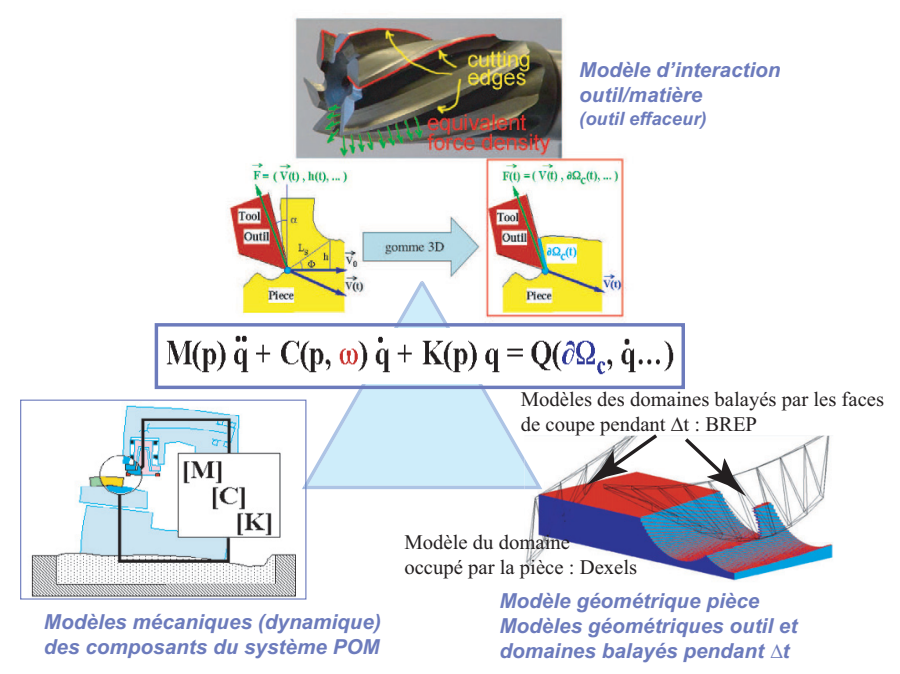

Fig. 1. Modèles utilisés pour la simulation.

Cette démarche prend en compte les variations significatives de masse et de rigidité. Des exemples de simulations en tournage et en fraisage sont présentés avant une courte conclusion.

\section{Modèles nécessaires}

La prédiction des efforts de coupe, et du comportement dynamique du système $\boldsymbol{P O M}$, passent ainsi par l'utilisation d'un modèle géométrique de la pièce et de sa surface usinée qui évolue au cours de temps et d'un modèle éléments-finis de la pièce et du reste du système $\boldsymbol{P O M}[2]$. Ces différents modèles sont illustrés de façon schématique sur la figure 1.

\subsection{Modèles éléments-finis et équation du mouvement}

Lorsque la pièce ne peut être considérée comme rigide, un modèle éléments-finis vient compléter sa description mécanique. Ici la pièce est modélisée par des éléments plaque. Le reste du système $\boldsymbol{P} \boldsymbol{O M}$ ne pose pas de problème spécifique. Il est généralement modélisé de façon simplifiée grâce à des systèmes masses/ressorts mais peut également faire intervenir, pour certaines de ses parties, des modèles éléments-finis : éléments poutres pour la broche, éléments coques ou tridimensionnels si nécessaire. Nous notons ${ }^{*} X$ les coordonnées du point courant dans les configurations de référence non déformées de l'outil et de la pièce avant bridage. Une discrétisation $U\left({ }^{*} X, t\right)=N\left({ }^{*} X\right) \cdot q(t)$ du champ de déplacement du système $\boldsymbol{P O M}$ permet de mettre en place un système matriciel (1) en décrivant le mouvement par l'intermédiaire des paramètres cinématiques $q$.

$$
\begin{gathered}
\boldsymbol{\psi}=0 \\
\boldsymbol{\psi} \stackrel{d}{=} \mathrm{M}(p) \ddot{q}+\mathrm{C}(p, \omega) \dot{q}+\mathrm{K}(p) q \\
-Q_{\text {coupe }}\left(q, \dot{q}, \partial \Omega_{\text {coupe }}, t\right)
\end{gathered}
$$

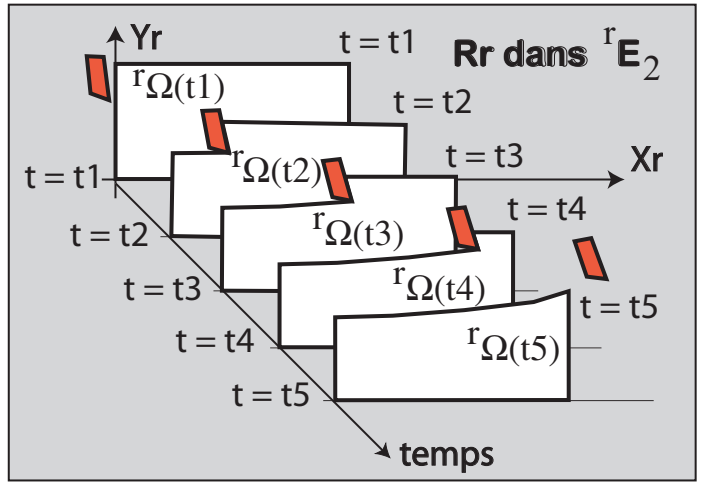

Fig. 2. Vue schématique, dans un cas bidimensionnel $\left({ }^{\mathrm{r}} E_{2}\right)$, de la notion d'outil effaceur de matière : évolution du domaine ${ }^{\mathrm{r}} \Omega(t)$.

Les composantes $q_{i}(t)$ de la matrice colonne ${ }^{t} q \equiv q(t)$ sont les inconnues du problème. Avec la mise en équations que nous adoptons, les matrices $\mathrm{M}(p), \mathrm{C}(p, \omega)$ et $\mathrm{K}(p)$ sont respectivement les matrices de masse, d'amortissement (intégrant éventuellement un effet gyroscopique dépendant de la vitesse de rotation $\Omega$ de la broche) et de rigidité construites en tenant compte de la géométrie donnée par $p$.

Malgré l'enlèvement de matière, dans de nombreux cas les matrices de masse et de rigidité peuvent être considérées comme constantes sur un intervalle de temps très largement supérieur au pas de temps retenu dans le schéma incrémental de résolution. La résolution de (1) est obtenue en utilisant un schéma (implicite) de Newmark utilisant un pas de temps constant $\Delta t[3]$.

\subsection{L'outil vu comme une gomme tridimensionnelle de matière}

Le second membre $Q_{\text {coupe, }}$ qui provient des efforts de coupe, introduit un terme non-linéaire dans (1). Ceci conduit à la mise en place d'un schéma itératif de résolution sur chaque incrément de temps. $Q_{\text {coupe }}$ est obtenu à partir du modèle d'interaction outil/pièce utilisé. Pour cette étude il se base :

- sur la notion d'outil effaceur de matière : là où les parties actives de l'outil passent la matière disparaît, ceci est illustré schématiquement sur la figure 2 ,

- sur une loi de coupe (dite « macroscopique») qui donne les efforts de coupe appliqués par l'outil sur la pièce, sous forme d'une densité linéïque $f_{\text {coupe }}$ en fonction des conditions instantanées de coupe.

La formulation éléments-finis permet d'en déduire $Q_{\text {coupe }}$ à chaque instant et la position « d'équilibre » associée, qui est donnée par $q$. Ce modèle d'interaction outil/pièce, relativement simple où les aspects thermomécaniques de la coupe ne sont pas explicitement pris en compte, permet néanmoins de générer une évolution réaliste de la surface usinée et des efforts de coupe $[4,5]$ à l'échelle considérée ici. Il est retenu de façon usuelle pour réaliser les études de 
stabilité de la coupe ou pour prendre en compte l'influence des déformations de l'outil dans les approches où la pièce est supposée parfaitement rigide

\subsection{Modèle d'interaction outil/pièce}

Pour prévoir le broutement il est nécessaire de suivre avec précision l'évolution des efforts de coupe. Ceci passe par une définition précise de l'effacement de la matière pour permettre, notamment, de prendre en compte les aspects régénératifs. Dans le cas d'une pièce déformable, ceci nécessite de définir un espace matériel de référence ${ }^{\mathrm{r}} E_{3}$ pour suivre, dans le détail, l'évolution de l'image ${ }^{\mathrm{r}} \Omega(t)$ du domaine [4]. Dans ${ }^{\mathrm{r}} E_{3}$, les images $s$ des points $X$ de la pièce, sont immobiles quelle que soit la configuration de la pièce. ${ }^{*} X$ désigne un point de la pièce dans sa configuration non bridée et fixée isostatiquement dans $\mathcal{R}_{\text {pièce }}$.

$\grave{A}$ chaque instant $t$, le domaine ${ }^{t} \Omega$ occupé par la pièce est suivi dans le référentiel de calcul $\mathcal{R}_{\text {pièce }}$ grâce à l'application bijective ${ }^{t} \Phi$ définie par (3) :

$$
\begin{aligned}
&{ }^{t} \Phi:{ }^{\mathrm{r}} \mathcal{B} \subset{ }^{\mathrm{r}} E_{3} \rightarrow{ }^{t} \mathcal{B} \subset E_{3}=\mathcal{R}_{\text {pièce }} \\
& s \mapsto{ }^{t} X={ }^{*} X+{ }^{\mathrm{t}} U \\
&{ }^{t} X={ }^{*} X+\mathrm{N}\left({ }^{*} X\right) \cdot{ }^{t} q \\
&{ }^{\mathrm{t}} \Omega(t) \subset{ }^{\mathrm{t}} \mathcal{B} \mapsto{ }^{t} \Omega \subset{ }^{t} \mathcal{B} \\
&{ }^{t} \Phi^{-1}{ }^{t} \mathcal{B} \subset E_{3}=\mathcal{R}_{\text {pièce }} \rightarrow{ }^{\mathrm{r}} \mathcal{B} \subset{ }^{\mathrm{r}} E_{3} \\
&{ }^{t} X \mapsto s
\end{aligned}
$$

Avant mise en place de la fixation de la pièce (avant bridage), la position de la pièce dans $\mathcal{R}_{\text {pièce }}$ est définie par une fixation isostatique. Le domaine ${ }^{*} \Omega$ occupé par la pièce $\mathcal{R}_{\text {pièce }}$ est défini par l'application bijective ${ }^{*} \Phi$ qui est choisie pour définir la géométrie de la pièce et des $\boldsymbol{D e x e l s}$ qui la décrivent. Les Dexels sont des parallélépipèdes rectangles jointifs de section carrée, associés à une grille et disposés suivant l'axe $Z$, figure 5 . La géométrie initiale de la pièce avant bridage et ${ }^{*} \Phi$ sont des données.

$$
\begin{aligned}
{ }^{*} \Phi: \quad{ }^{\mathrm{r}} \mathcal{B} \subset{ }^{\mathrm{r}} E_{3} & \rightarrow{ }^{*} \mathcal{B} \subset E_{3}=\mathcal{R}_{\text {pièce }} \\
s & \mapsto{ }^{*} X \\
{ }^{\mathrm{r}} \Omega(0) \subset{ }^{\mathrm{r}} \mathcal{B} & \mapsto{ }^{*} \Omega \subset{ }^{*} \mathcal{B} \\
{ }^{*} \Phi^{-1}:{ }^{*} \mathcal{B} \subset E_{3}=\mathcal{R}_{\text {pièce }} & \rightarrow{ }^{\mathrm{r}} \mathcal{B} \subset{ }^{\mathrm{r}} E_{3} \\
{ }^{*} X & \mapsto s
\end{aligned}
$$

Afin de pouvoir ramener dans ${ }^{\mathrm{r}} E_{3}$ les faces de coupe de l'outil, puis de pouvoir y construire le domaine balayé par ces surfaces pendant un incrément de temps $\Delta t$, ces applications doivent ensuite être prolongées à l'extérieur de ${ }^{r} \mathcal{B}$. Ceci est précisé dans la suite (Fig. 6).

La figure 3 montre le prolongement ${ }^{t} \Phi^{+}$de ${ }^{t} \Phi$ qui est précisé plus loin pour une pièce très voisine de celle considérée dans l'exemple de simulation réalisée en fraisage. ${ }^{t} \Phi$ est confondue avec ${ }^{t} \Phi^{+}$pour tout point du domaine pièce ${ }^{r} \Omega(0)$.

La bijection de travail ${ }^{*} \Phi: s \mapsto{ }^{*} X$ retenue dans cette présentation est une isométrie comme c'est le cas sur la figure 3. La figure 6 illustre de façon schématique

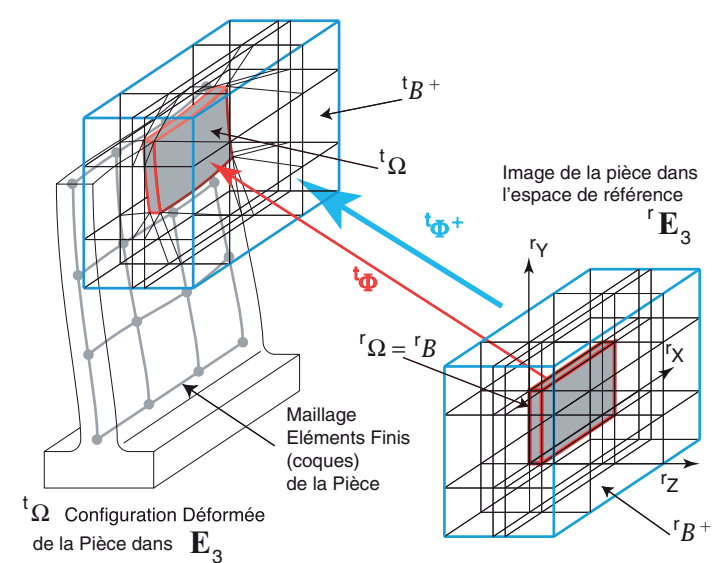

Fig. 3. Application ${ }^{t} \Phi^{+}$, cas où ${ }^{*} \Phi$ est une isométrie et où le calcul par éléments-finis est réalisé avec des éléments de coque. ${ }^{\mathrm{r}} \Omega(0)$ et ${ }^{t} \Omega$ sont représentés en grisé sur cette figure.

cette transformation dans le cas bidimensionnel lorsque la transformation est quelconque (ceci permet d'orienter les Dexels de façon à maximiser la qualité de la représentation par rapport aux opérations d'usinage).

Le domaine initial $* \Omega$ de la pièce usinée (en réalité la partie usinée de ce domaine suffit), dans sa configuration de référence est supposé entièrement contenu dans ${ }^{*} \mathcal{B}$ ou confondu avec ${ }^{*} \mathcal{B}$. Les autres transformations ${ }^{t} \Phi$ se représenteraient de façon analogue, ${ }^{*} \mathcal{B}$ et ${ }^{*} \Omega$ se transformant alors en ${ }^{t} \mathcal{B}$ et ${ }^{t} \Omega$. C'est le déplacement ${ }^{t} U$ qui permet de les obtenir à partir du choix de ${ }^{*} \Phi$.

La condition initiale ${ }^{0} q$ est obtenue par une résolution statique associée au bridage et donne ${ }^{0} \Phi$.

\subsection{Modèle donnant les efforts de coupe}

Dans les approches à l'échelle macroscopique, les efforts de coupe sont modélisés via des densités linéïques

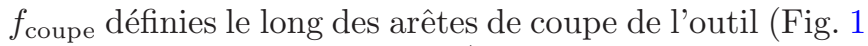
et la partie droite de la Fig. 4). Des modes de construction de modèles donnant les composantes de $f_{\text {coupe }}$ dans des repères liés aux faces de coupe sont disponibles. Par exemple, dans [6] la notion de couple arête-matière permet de construire efficacement ce type de modèle en le rendant utilisable pour un ensemble de couples outil/matière.

Ces modèles font en général intervenir $h_{\text {coupe }}$, l'épaisseur locale instantanée de coupe et la vitesse relative locale outil/pièce. $h_{\text {coupe }}$ est obtenue, pour chaque partie de face de coupe par des considérations géométriques, en partant du volume de matière enlevée et en effectuant une moyenne sur $\Delta t$. Ceci permet un lissage de l'évaluation de $f_{\text {coupe }}$ qui améliore la convergence.

\subsection{Modèles géométriques}

Dans le contexte d'un schéma temporel incrémental, la modélisation de l'enlèvement de matière et de l'interaction instantanée outil/pièce nécessitent de mettre en 


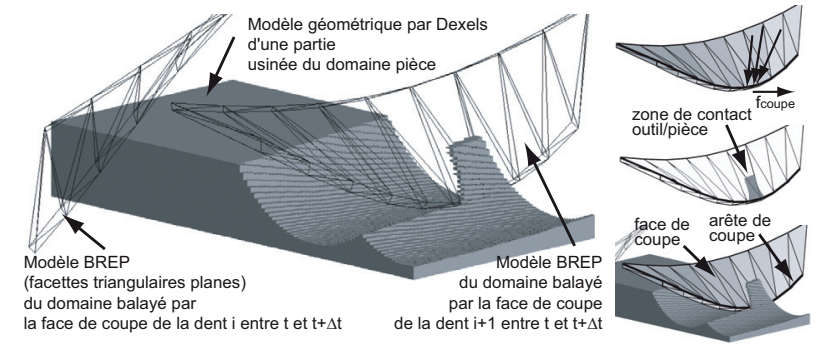

Fig. 4. Modèles géométriques dans l'espace de référence matériel : pièce (Dexels axe $Z$ vertical sur la figure) et domaine balayé par les faces de coupe des dents $i$ et $i+1$ d'une fraise hélicoïdale dans ${ }^{\mathrm{r}} E_{3}(\boldsymbol{B R} \boldsymbol{E P})$.

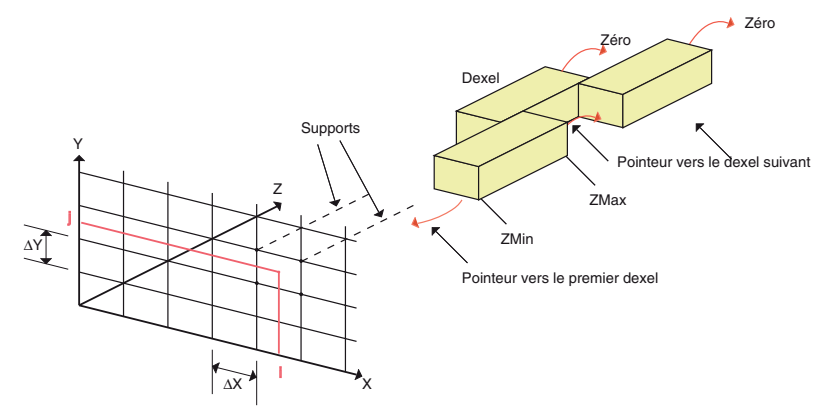

Fig. 5. Principe de construction dans ${ }^{\mathrm{r}} E_{3}$ d'un modèle géométrique utilisant des Dexels.

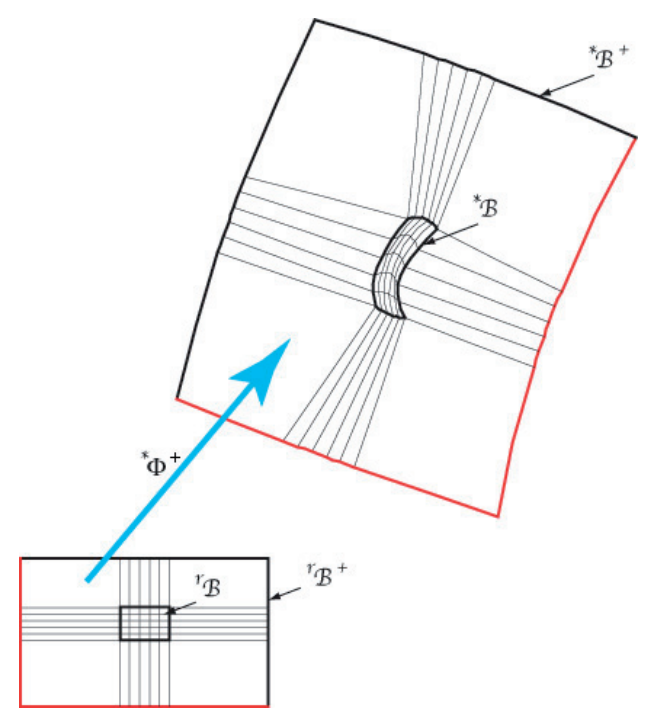

Fig. 6. Bijection ${ }^{*} \Phi^{+}$entre l'espace de référence ${ }^{\mathrm{r}} E_{3}$ et l'espace $E_{3}$ associé à $\mathcal{R}_{\text {pièce }}$ pour définir ${ }^{*} \Omega$, configuration de référence de la pièce.

place deux modèles géométriques : le modèle $\mathcal{M}_{\mathrm{b}}$ des domaines balayés par les faces de coupe pendant l'incrément de temps $\Delta t$, et celui du domaine évolutif de la pièce. Pour les domaines balayés une représentation par leur frontière (modèle $\boldsymbol{B R E P}$ pour Boundary REPresentation) composée de facettes triangulaires planes a été retenue. Le domaine évolutif de la pièce est quant à lui, comme précisé plus haut, représenté par des $\boldsymbol{D}$ exels dans ${ }^{\mathrm{r}} E_{3}$. Ils permettent de modéliser ${ }^{\mathrm{r}} \Omega(t)$ à mesure que l'effacement de matière se produit. Ce dernier choix permet des traitements optimisés des algorithmes d'intersection.

La nécessité de réaliser les intersections dans ${ }^{\mathrm{r}} E_{3}$ impose par ailleurs de transporter à chaque itération de chaque incrément les sommets des facettes de $\mathcal{M}_{\mathrm{b}}$ depuis l'espace réel $E_{3}$ associé à $\mathcal{R}_{\text {pièce }}$.

Pour les sommets appartenant au maillage de calcul c'est immédiat (mais itératif) via ${ }^{t} \Phi^{-1}$. Pour les autres sommets il est nécessaire de construire une extension ${ }^{t} \Phi^{+}$ de ${ }^{t} \Phi$ : ceci est réalisé via un maillage hexaédrique qui est défini de façon régulière à l'intérieur des éléments de plaque qui occupent un domaine ${ }^{*} \mathcal{B}$, et étendu dans un domaine parallélépipédique (rectangle) de très grande taille à l'extérieur ${ }^{*} \mathcal{B}^{+}$. La figure 6 illustre cette façon de faire de façon schématique : la représentation est bidimensionnelle pour simplifier et les boîtes externes ${ }^{r} \mathcal{B}^{+}$et ${ }^{*} \mathcal{B}^{+}$ne sont pas représentées à l'échelle.

Une matrice de liaison $G_{\text {calcul }}^{\text {hexa }}$ permet de réaliser un transfert approché du champ $U$ en fixant grâce à lui les déplacements $q_{\text {hexa }}$ des nouds des hexaèdres définissant ${ }^{t} \Phi^{+}: q_{\text {hexa }}=\mathrm{G}_{\text {calcul }}^{\text {hexa }} \cdot q$. Les déplacements des nœuds très éloignés sont fixés à 0 .

$$
\begin{aligned}
& { }^{t} \Phi^{+} \text {: } \\
& { }^{\mathrm{r}} \mathcal{B}^{+} \subset{ }^{r} E_{3} \rightarrow{ }^{t} \mathcal{B}^{+} \subset E_{3}=\mathcal{R}_{\text {pièce }} \\
& s \mapsto{ }^{t} X={ }^{*} X+{ }^{t} U_{\text {hexa }} \\
& { }^{t} U_{\text {hexa }}=\mathrm{N}_{\text {hexa }}\left({ }^{*} X\right) \cdot{ }^{t} q_{\text {hexa }} \\
& { }^{\mathrm{r}} \Omega(t) \subset{ }^{\mathrm{r}} \mathcal{B} \mapsto{ }^{t} \Omega \subset{ }^{t} \mathcal{B}
\end{aligned}
$$

La détermination du domaine balayé par les faces de coupe pendant l'intervalle de temps $] t, t+\Delta t]$ correspondant à un pas de temps du schéma d'intégration de Newmark nécessite un sous-découpage pour obtenir une description correcte dans le cas du fraisage. Sinon, les vitesses de rotation de broche importantes conduisent à des erreurs géométriques trop importantes. On réalise donc une linéarisation utilisant $n_{\text {int }}$ sous-incréments en supposant des trajectoires rectilignes des sommets dans ${ }^{\mathrm{r}} E_{3}$ pendant chaque sous intervalle de durée $\Delta t / n_{\text {int }}$. Toutes les positions intermédiaires doivent être ramenées dans ${ }^{\mathrm{r}} E_{3}$ à chaque itération $i$ de chaque incrément pour évaluer les estimations $i$ des domaines effacés ainsi que celles du domaine actuel ${ }^{t} \Omega^{(i)}$ et de sa frontière ${ }^{t} \partial \Omega^{(i)}$.

\subsection{Prise en compte des pertes de matière}

Lorsqu'elles ne sont pas négligeables, les conséquences des pertes de masse et de rigidité sont prises en compte de façon simplifiée en utilisant la connaissance a priori que l'on a de la trajectoire «théorique outil/pièce » et par suite de $p(t)$. Ceci conduit à un schéma de résolution qui ne nécessite la mise à jour des matrices de rigidité et de masse qu'au passage de certains piquets de temps $T_{j}$ définis à l'avance. La méthode utilise une interpolation linéaire de l'inverse de l'opérateur de Newmark entre deux piquets [4]. Les différents modèles éléments-finis de la pièce associés à chaque piquet de temps retenu pour les changements de matrices peuvent être construits et 


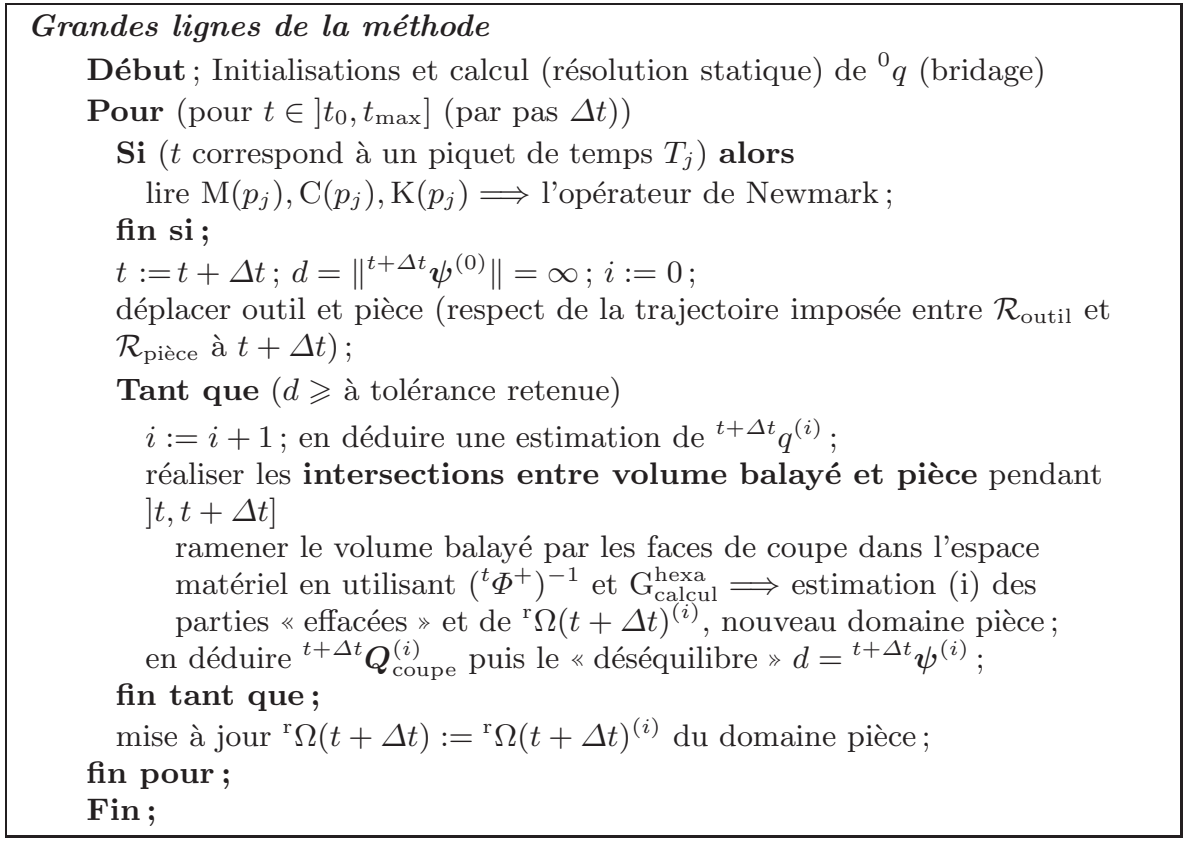

les matrices calculées et stockées avant le lancement de la simulation.

\section{Démarche adoptée}

La démarche est détaillée dans [2]. Les différents modèles éléments-finis de la pièce associés à chaque piquet de temps retenu pour les changements de matrices EF peuvent avoir été préparés (en réalisant éventuellement des condensations statiques) au préalable et les matrices correspondantes stockées, ainsi que les opérateurs de Newmark et leurs décompositions de Cholesky ou autre.

L'initialisation du processus de calcul comprend les étapes suivantes :

- lecture du modèle géométrique de l'outil défini dans $\mathcal{R}_{\text {outil }}$,

- lecture du modèle géométrique la pièce défini dans $\mathcal{R}_{\text {pièce }}$,

- lecture du maillage EF de la pièce défini dans $\mathcal{R}_{\text {pièce }}$,

- construction de l'opérateur $\mathrm{G}_{\text {calcul }}^{\text {hexa }}$,

- lecture des conditions de liaison de la pièce dans $\mathcal{R}_{\text {pièce }}$ (bridage),

- lecture des modèles de calcul de $f_{\text {coupe, }}$

- lecture des trajectoires imposées entre $\mathcal{R}_{\text {outil }}$ et $\mathcal{R}_{\text {pièce }}$.

La détermination de $\mathrm{G}_{\text {calcul }}^{\text {hexa }}$ est effectuée une fois pour toute avant le début de la simulation. Il s'agit simplement de conserver, pour chaque degré de liberté d'un nœud du maillage en hexaèdres, la combinaison linéaire des degrés de liberté de $q$ permettant de la calculer. Ceci passe par l'utilisation de la cinématique de la théorie des coques (plaques dans les exemples retenus). Compte tenu de la position assignée à chaque degré de liberté dans $q_{\text {hexa }}$, on en déduit immédiatement le contenu de $\mathrm{G}_{\text {calcul }}^{\text {hexa }}$.

\section{Exemples}

Dans les deux exemples présentés, le nombre des Dexels qui est indiqué sur les figures correspond uniquement à la description du modèle géométrique de la partie usinée de la pièce. En effet, seule cette partie usinée de la pièce nécessite une description géométrique fine de ce type, afin de réaliser la simulation. Ceci n'est pas été détaillé par soucis de concision.

\subsection{Fraisage}

L'exemple du fraisage d'une portion d'une pièce mince permet de mettre en évidence les différences qui apparaissent sur la surface usinée finale en fonction du rapport $f_{\mathrm{d}} / f_{1}=\frac{\text { fréquence de passage des dents }}{\text { fréquence propre } 1 \text { de la pièce }}$. Ce type de pièce, qui a été conçu au $\boldsymbol{L} \boldsymbol{M S P}$ par François Lapujoulade [7], permet de mettre en évidence et d'observer finement ces phénomènes de façon expérimentale.

Les défauts prédits en faisant l'hypothèse que la pièce est indéformable ne sont pas visibles (Fig. 7a) et correspondent aux défauts prédits par les modèles purement cinématiques de l'usinage tels qu'on les trouve dans les modeleurs géométriques classiques. Une fréquence de passage des dents égale à la première fréquence propre de vibration de la pièce conduit par contre déjà à des défauts géométriques significatifs (Fig. 7c).

Cependant, ces défauts sont sans commune mesure avec ceux que l'on peut mettre en évidence sous l'action d'une excitation résultant d'une fréquence de rotation de la broche conduisant à un rapport $f_{\mathrm{d}} / f_{1}$ voisin de 0,515 (Fig. 7b). Ce défaut extrêmement important par rapport aux deux autres montrés apparaît à cause du couplage entre le comportement vibratoire de la pièce et la fluctuation des efforts de coupe qui en résultent. 


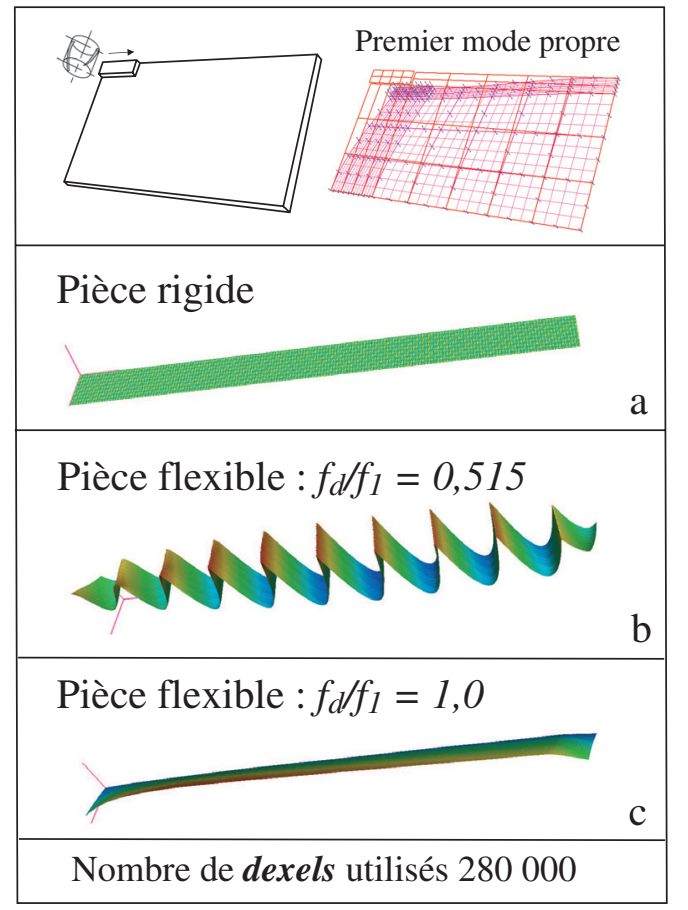

Fig. 7. Surface finale simulée en fraisage : dynamique transitoire, diverses vitesses de rotation.

Les géométries de surface calculées dans les 3 simulations sont affichées en effectuant les tracés avec un coefficient multiplicateur identique de 10 (suivant la normale à la surface théorique).

Pour les exemples traités, le choix du nombre de Dexels est directement lié au choix du pas de temps et à la valeur de la vitesse de coupe : balayage d'au moins 5 Dexels dans la direction de la vitesse de coupe pour limiter les oscillations dues à la description discrète du volume effacé pour l'estimation des efforts de coupe. Il est à préciser que le nombre total de Dexels coupés sur un pas de temps dépend également de la largeur de coupe et que les fluctuations de l'effort de coupe dépendent également de l'orientation de la vitesse de coupe vis-à-vis de la matrice des supports de Dexels. Le choix du pas de temps est quant à lui directement lié à la plus grande fréquence $f_{\text {Max }}$ que l'on souhaite observer avec fidélité : $\Delta t \approx \frac{15}{f_{\text {Max }}}$.

Pour cet exemple la durée de simulation est d'environ 15 minutes sur un serveur de calcul Hewlett Packard.

\subsection{Tournage}

L'exemple de tournage présenté figure 8 illustre les possibilités de l'approche proposée pour une simulation en statique d'une pièce industrielle (SNECMA-SAFRAN).

Le bridage réalisé à l'aide de 8 brides réparties sur la circonférence de la pièce (disque de diamètre $0,6 \mathrm{~m}$ ) déforme la pièce par rapport à sa forme initiale. Cette déformation est donnée par ${ }^{*} \Phi$ au début de l'opération. Ceci est la principale cause des creux observés sur la surface usinée finale. La figure 8 montre le découpage du

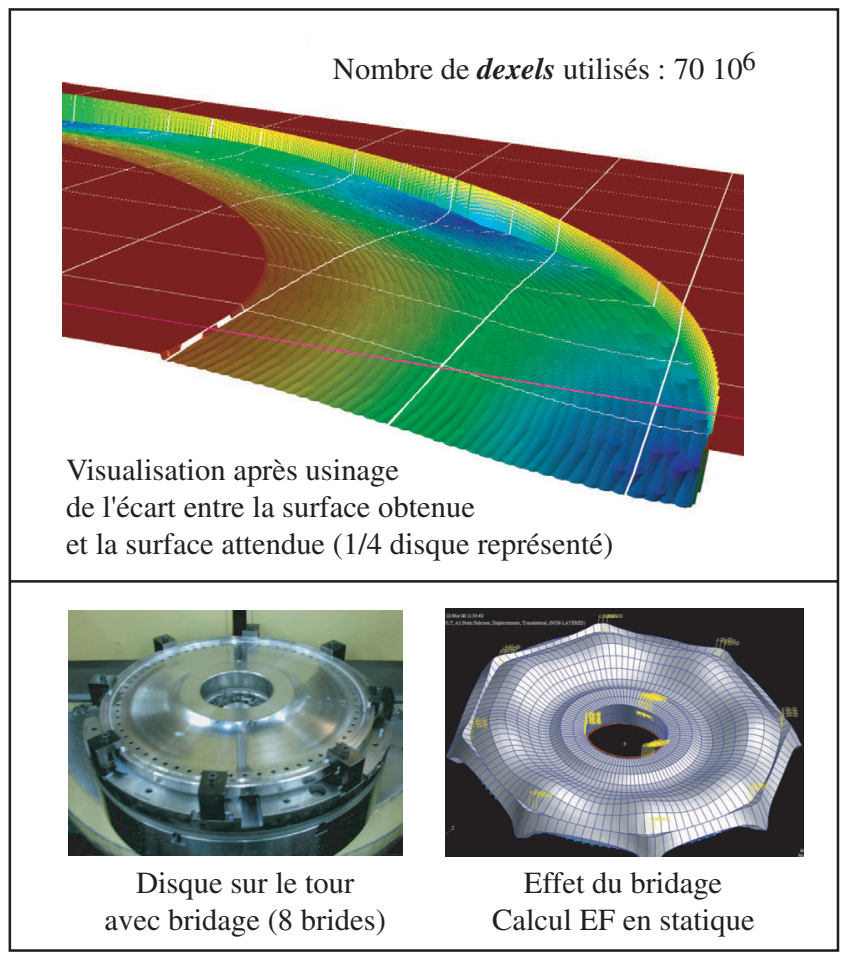

Fig. 8. Surface finale simulée en tournage : calcul statique avec bridage.

modèle Dexels en patchs. Ce découpage en «patchs » est associé à une gestion informatique élaborée qui utilise un stockage paginé du modèle géométrique Dexels. Ceci permet de traiter des modèles géométriques de taille quelconque tout en conservant une bonne efficacité numérique. Pour cet exemple la durée de simulation est d'environ 1 heure sur un serveur de calcul Hewlett Packards ${ }^{1}$.

\section{Conclusion}

La simulation numérique de l'usinage de pièces flexibles est un problème non encore complètement résolu. La démarche proposée et les diverses modélisations proposées permettent d'envisager la possibilité de mettre en place à relativement court terme des logiciels métier permettant d'apporter une réponse en milieu industriel.

La démarche présentée ici est actuellement implantée dans $\boldsymbol{N e s s y}$ le logiciel développé au $\boldsymbol{L} \boldsymbol{M S P}$ pour concevoir et tester des méthodes de résolution nouvelles. Les deux exemples présentés ont simplement pour objectif de montrer que, d'une part la prise en compte de la flexibilité de la pièce est indispensable pour être sûr de ne pas passer à côté de phénomènes vibratoires essentiels, d'autre part que l'approche peut s'appliquer dans le cas beaucoup plus simple des comportements quasi statiques. Par ailleurs, le

\footnotetext{
1 Configuration : 2 processeurs Xeon double cœurs cadencés à 3,2 GHz - Mémoire cache : 2 MegaOctets/processeur Mémoire vive : 8 GigaOctets
} 
second exemple proposé montre la capacité de l'approche à traiter des pièces industrielles pour lesquelles l'étendue de la surface usinée est grande.

Des simulations étant maintenant possibles, il va être nécessaire de valider plus finement les résultats obtenus par comparaison à des résultats expérimentaux.

L'approche proposée ici ne concerne que l'usinage dans des zones où la pièce peut être modélisée par la théorie des coques. Sans changer profondément les algorithmes, cette approche peut être adaptée pour des usinages dans des zones quelconques de pièces complexes en s'affranchissant de l'utilisation de la théorie de coques. C'est un de nos objectifs à court terme.

Remerciements. Nous tenons à remercier MM. C. Le Calvez, J.-F. Chabot et A. Perez-Duarte (SNECMA-SAFRAN) pour leur soutien à cette étude qui nous a permis, en particulier, de vérifier les capacités de la méthode sur une pièce de grande taille en tournage.

\section{Références}

[1] H.E. Merritt, Theory of self-excited machine tool chatter - resarch I, J. Eng. Ind. 17 (1965) 447-454
[2] S. Cohen-Assouline, Simulation numérique de l'usinage à l'échelle macroscopique : prise en compte d'une pièce déformable, Ph.D. Thèse, École Nationale Supérieure d'Arts et Métiers, décembre 2005

[3] G. Coffignal, E. Beauchesne, K. Dekelbab, N. Hakem, Mechanical simulation of machining, ed. P. Chedmail, J.C. Bocquet, \& D. Dornfeld, IDMME'97, Kluwer Academic, 1997, pp. 297-306

[4] S. Jayarama, S. Kapoor, R.E. DeVor, Estimation of the specific cutting pressures for mechanistic cutting force models, Int. J. Machine Tools Manufacture 41 (2001) 265-281

[5] F. Atabey, I. Lazoglu, Y. Altintas, Mechanics of boring processes - Part I, Int. J. Machine Tools Manufacture 43 (2003) 463-476

[6] S. Bissey, Développement d'un modèle d'effort de coupe applicable à des familles d'outils : cas du fraisage des aciers traités thermiquement, Ph.D. Thèse, École Nationale Supérieure d'Arts et Métiers, avril 2005

[7] F. Lapujoulade, HDR : Simulation des Phénomènes Vibratoires Intervenant en Usinage : État des recherches et perspectives, Ph.D. Thèse, Institut National des Sciences Appliquées de Lyon et Université Claude Bernard Lyon I, mai 2003

[8] P. Lorong, HDR : Apport du calcul de structures dans la modélisation numérique de l'usinage : Échelle macroscopique - Échelle mésoscopique, Ph.D. Thèse, Université Pierre et Marie Curie, Paris 6, décembre 2006 